\title{
Battle of the mind
}

\section{Plans for a vaccine for Alzheimer's disease were derailed last year when clinical trials revealed serious side-effects. But as Erika Check finds out, this approach could be about to get back on track.}



Anatomy of dementia: tissue damage and death in a brain from an Alzheimer's patient (left) leads memory loss and changes in personality.

$\mathrm{T}$ hree years ago, a 72-year-old British woman with Alzheimer's disease became one of the first people in the world to test a promising experimental treatment for her condition. Starting in July 2000, doctors gave her and around 70 other patients doses of a vaccine designed to prompt their immune systems to attack and clear away the clumps of proteins that many believe cause the symptoms of Alzheimer's disease. Researchers were cautious, but hopes were high that the vaccine, which contained a form of the $\beta$-amyloid protein found in the plaques, could treat this distressing disease.

After promising results from this small safety trial, the pharmaceutical company behind the vaccine - Elan, in San Francisco, California - launched a larger trial the following year. But by the summer of 2001, problems began to emerge. The British woman was suffering from dizzy spells and having trouble walking. In January 2002, Elan pulled the plug on its second trial after patients showed signs of brain inflammation. The woman from the original trial died of a blood clot a month later. Hopes for the vaccine seemed to be dead in the water.

\section{Take two}

But not quite. The post mortem on the British woman, carried out by neuropathologist James Nicoll of the University of Southampton, UK, and published this month ${ }^{1}$, reveals that the treatment probably cleared some protein clumps. The first vaccine may have been unsafe, but many Alzheimer's researchers believe this approach could still produce a cure. Later this year, tests on a second-generation Alzheimer's vaccine could get under way. "Alzheimer's disease is devastating," says Dale Schenk, who led the Elan team that developed the vaccine. "If you look at the data there's more evidence for this approach being potentially efficacious than most others."

The idea of developing an immunotherapeutic treatment for Alzheimer's emerged in the late 1990s. The notion was based on the amyloid hypothesis, the theory that misfolded clumps of a certain protein, known as $\beta$-amyloid, cause the symptoms of Alzheimer's. Elderly people commonly have clumps of $\beta$-amyloid, known as plaques, in their brains. But Alzheimer's sufferers have many more, and eliminating or modifying the plaques is now the focus of much Alzheimer's research.

In 1999, Schenk and his colleagues produced some of the earliest concrete evidence in favour of using the immune system to do this ${ }^{2}$. Working with transgenic mice that had been genetically modified to make human $\beta$-amyloid, they showed that these animals 
developed fewer plaques if they were injected early in life with $\beta$-amyloid proteins. Even more intriguingly, they also found that injecting the protein cleared away plaques that had already formed in the brains of older mice.

Schenk and his colleagues thought that they knew roughly what was happening. They reasoned that the mice were treating the injected $\beta$-amyloid protein like an invading pathogen and generating antibodies against it. These antibodies then attached themselves to the $\beta$-amyloid and recruited other cells to eliminate the plaques. The cells they recruited - small immunesystem cells known as microglia - chewed up and digested the plaques, leaving healthy brain cells behind.

The technique, known as active vaccination, was considered enormously promising. "Everybody got excited, thinking this was going to be a cure," says David Holtzman, a neuroscientist at Washington University in St Louis, Missouri. "They were rightfully excited - it was a very dramatic effect."

\section{Antibody alternatives}

Over the next three years, neuroscientists learned a lot about Alzheimer's vaccines. Two groups immunized similar transgenic mice with the $\beta$-amyloid and ran the animals though a water maze designed to test their memory skills. Vaccinated mice performed better than unvaccinated mice as the two groups aged ${ }^{3,4}$. The vaccine not only cleared plaques - it also seemed to be lessening the symptoms of the disease.

Meanwhile, a related immunotherapeutic approach was producing promising results at Elan's San Francisco labs. In 2000, Frédérique Bard and colleagues showed that rather than injecting mice with the $\beta$ amyloid protein, they could clear away brain plaques by injecting the animals with antibodies against the $\beta$-amyloid ${ }^{5}$. And last year, Holtzman and colleagues, together with a team from drug giant Eli Lilly in Indianapolis, Indiana, showed that this approach, known as passive vaccination, staves off learning and memory loss in transgenic mice that normally develop an Alzheimer'slike disease ${ }^{6}$.

The outlook for both approaches was rosy. None of the mouse studies reported side effects and Schenk says that unpublished primate studies had indicated that the $\beta$-amyloid-based vaccine was safe. Opting for the active approach — the better developed of the two - Elan started its first human safety trials with this vaccine in 2000, which included the woman later studied by Nicoll. Initial unpublished results from this trial were good enough for the US Food and Drug Administration (FDA) to give the go-ahead in 2001 for a larger trial of 375 sufferers in the United States and Europe, designed to find out whether the vaccine



Plaques multiply in the Alzheimer's brain (left).

would alleviate their symptoms.

But not everyone was convinced the trials were a good idea. $\beta$-amyloid collects in blood vessels in the brain, and an attack on these could cause brain haemorrhages. Another problem could come from a second line of immunological defences in which the immune system damages large areas around target proteins. That could be disastrous in the brain, as brain cells are unlikely to be replaced.

To make matters worse, $\beta$-amyloid is made from a larger precursor protein, which is sprinkled liberally throughout the brain. There was a theoretical chance that immune cells targeted at $\beta$-amyloid would recognize and destroy the precursor protein and any normal cells it was attached to. "You are vaccinating with something you already know is toxic, and which could create an autoimmune disease and inflammation," says Blas Frangione, a neuroscientist at New York University. "We thought it was not a good idea."

These doubts soon appeared justified. The second trial was halted when worrying signs of brain inflammation showed up in $6 \%$ of patients. And when the brain of the woman from the first trial was examined by Nicoll, T cells - an immune-system component that can cause inflammation - were found in the tissue surrounding her brain, suggesting that the vaccine had prompted a harmful immune response. Nicoll believes the same mechanism caused the inflammation in the larger trial.

But amid the bad news, evidence emerged that the vaccine was working. Last October, Roger Nitsch and colleagues at the University of Zurich in Switzerland showed how blood from 24 Swiss patients in the larger trial contained antibodies against $\beta$-amyloid ${ }^{7}$. Nicoll's autopsy also hinted that these antibodies might be clearing protein clumps, as that patient's brain lacked plaques in the temporal lobe, a region that is usually heavily affected in Alzheimer's patients.

Furthermore, the brain areas that lacked plaques also lacked certain kinds of abnormalities seen in brain cells near plaques, even though these areas were still marked by other characteristics of Alzheimer's disease, such as $\beta$-amyloid in the blood vessels. Nicoll also found microglia, some containing fragments of $\beta$-amyloid, in the areas that lacked plaques.

For Schenk, the results are enough to suggest that the plaques were removed by the microglia. But other scientists are not so sure. The autopsy results have one major flaw: there was no way to examine the patient's brain before she received the vaccine, so it is impossible to prove that plaques were cleared.

There are other problems. One region of her brain - the frontal lobe - had the load of plaques usual in Alzheimer's patients. And Frangione says that a subset of Alzheimer's patients do not have plaques throughout their brains, and that the plaque distribution in the woman matched that of this subset. "We know there is a subgroup of patients who have very few plaques," he says. "So the authors should not conclude that clearance of the plaques is due to the treatment."

\section{Less is more}

Nicoll disputes this, pointing out that the pattern of plaques is also similar to that seen in the mouse models. The issue is unlikely to be settled without more data. Elan may release results of the cognitive tests they ran on patients in the second trial later this year, and Schenk hopes to find some evidence of efficacy. But regardless of these results, there are enough hints that the vaccine may work for neuroscientists to want to continue with this approach.

The issue now is how to proceed safely. In the past year, the field has focused on two main options - modifying the $\beta$-amyloid protein used to make the vaccine, and running further studies of passive immunization.

The entire $\beta$-amyloid protein contains 42 amino acids, and Elan gave patients the whole protein. But the complete protein may not be needed to provoke an immune response. And because one end of the protein is thought to stimulate the antibody response and the other end the T-cell response, it may be that a vaccine that contained a small part of the protein would elicit a wholly helpful immune reaction.

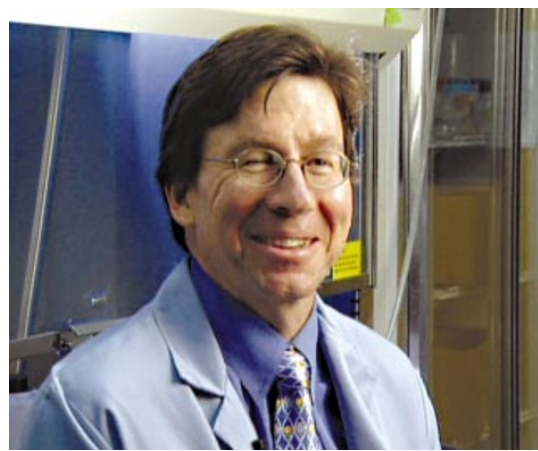

Still optimistic: Dale Schenk believes that an effective Alzheimer's vaccine can be developed. 
Last year, neuroscientists Peter St George-Hyslop and Joanne McLaurin at the University of Toronto vaccinated transgenic mice with a small chunk of $\beta$-amyloid containing seven acids from the end of the protein that prompts the antibody response, and found that this generated antibodies that recognized the complete $\beta$-amyloid protein ${ }^{8}$. These antibodies destroyed $\beta$ amyloid clumps in a culture dish, indicating that they might recognize $\beta$-amyloid plaques in animals, although McLaurin has not yet assessed this.

But would such an approach be safer? McLaurin tested the kinds of immune response that mice launched against the short version of $\beta$-amyloid. She found that they responded to the vaccine by producing antibodies that are unlikely to activate $T$ cells and that the animals did not produce the chemical signs that usually warn of inflammation. "All of this suggests that maybe by modifying the vaccine, you might be able to circumvent some of the inflammation," says McLaurin. Her group is now trying to find a small molecule that will mimic the effect of the limited chunk of $\beta$-amyloid. Small molecules are more attractive than macromolecules such as antibodies, as they are cheaper to make and easier to control.

\section{Passive potential}

Elan, in conjunction with the pharmaceutical company Wyeth of Madison, New Jersey, is also working on a vaccine based on a subunit of the $\beta$-amyloid protein. Even though the company was stung by the sideeffects in its previous trial, Schenk says that it is important to go ahead with new active immunization trials, and Elan plans to ask the FDA for clearance to start new studies later this year.

Like last time, some researchers are advising against clinical trials. Giving a dose of vaccine that activates microglia in the brain is a dangerous proposition, argues Michael Mullan, director of the Roskamp Institute for Research into Neurodegenerative Diseases in Tampa, Florida, "It's like giving a baby a loaded gun," he says. "If you switch the microglia on, you're really not in control of what happens."

Concerns such as these are one of the reasons why scientists began experimenting with passive immunization. Now, many say that this option looks more attractive than the active approach. Elan wants to start a second trial later this year to test whether it is safe to inject antibodies directly into patients' bloodstreams. The reasoning is simple: the antibodies should cross into the patients' brains, then recognize $\beta$-amyloid, bind to it, and stimulate a microglial response - all without involving T cells.

But the story may be more complicated. It's not easy for large proteins such as antibodies to cross the blood-brain barrier. In

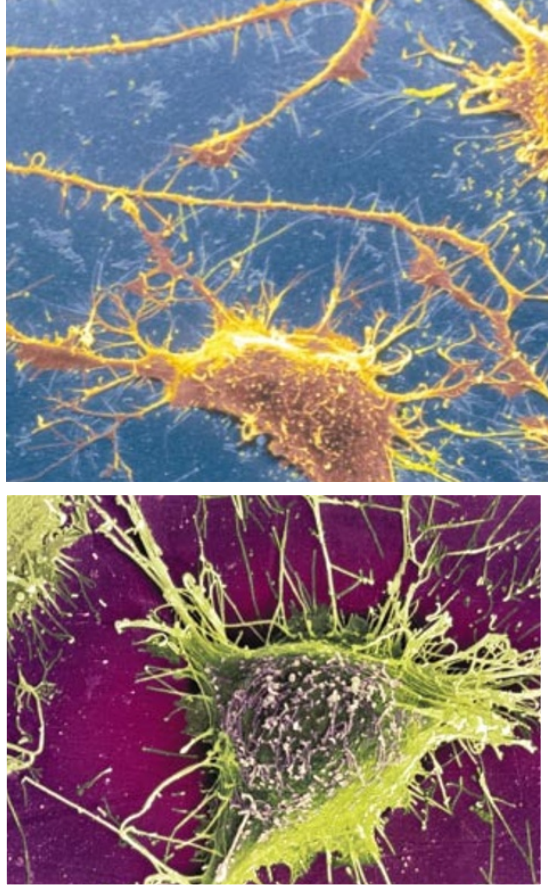

2001, Holtzman described how antibodies against $\beta$-amyloid may help clear away plaques without ever entering the brain. His team showed that injecting the antibodies into transgenic mice altered the levels of the protein circulating in the blood and the cerebrospinal fluid, which surrounds the brain 9. This prompted Holtzman to develop his 'peripheral sink' hypothesis: if $\beta$-amyloid is constantly circulating from the brain out into the cerebrospinal fluid and blood, removing it from the blood would eventually cut levels in the brain. This could explain why passive vaccination was able to clear away brain plaques even though few antibodies are able to cross from the blood into the brain.

The hypothesis appears to be backed up by a study published this January by Karen Duff, a neurologist at the Nathan Kline Institute for Psychiatric Research in Orangeburg, New York. Duff and colleagues found that small molecules constructed to bind and remove $\beta$-amyloid from the bloodstream can clear the protein out of the brains of transgenic mice ${ }^{10}$. "This would mean we don't have to use antibodies any more," says Duff. "We could use a non-toxic binding compound to clear $\beta$-amyloid from the blood and we wouldn't even need to go into the brain, which is a big advantage to pharmaceutical companies."

Before such ideas can be evaluated, researchers will have to convince regulators that immunotherapeutic approaches are safe enough for clinical trials. The problems with Elan's earlier studies still cast a long shadow over Alzheimer's research. "The sad thing was that Elan used active immunization rather than passive, and many scientists had warned that this would be the result," Duff says. "I think the FDA will be looking much more carefully at any potential treatments in the future because this one had such disastrous results."

Passive immunization may appear a safer
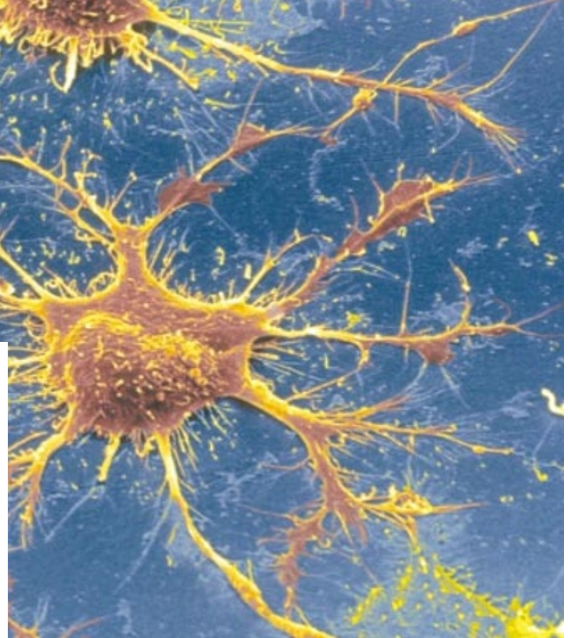

Cell help: nerve cells producing $\beta$-amyloid can be used to produce plaques in culture.

bet, but there are hints that this approach may also have problems. Frangione points out that the human body could potentially respond to antibody injections by simply making antibodies against the antibodies. And researchers at University of Basel in Switzerland reported last year that passive vaccination of mice elicited small haemorrhages in blood vessels in the brain that were loaded with $\beta$-amyloid ${ }^{11}$. Nobody has yet tested passive immunization in people, so it's hard to say whether the same effect would occur.

If the regulators do give their approval, the trials should also provide the answer to the crucial question of whether the $\beta$-amyloid hypothesis, backed by many Alzheimer's researchers for the past decade, is in fact correct. Even if the vaccine proves to be unsafe, the results of the clinical trials could make or break the theory. If the plaques are cleared, but the distressing memory loss and cell death that mark the disease remain, neuroscientists will be left wondering what it all means.

"This is the ultimate test of the amyloid hypothesis," says Nicoll. "It's a very important question, and it looks like we might be able to get a handle on it in the next year." If Elan's trials show that its vaccine does not work, it could do more than end hopes for the immunotherapy approach — it might also send scientists back to the drawing board to come up with a new theory of Alzheimer's disease.

\section{Erika Check is Nature's Washington biomedical}

\section{correspondent.}

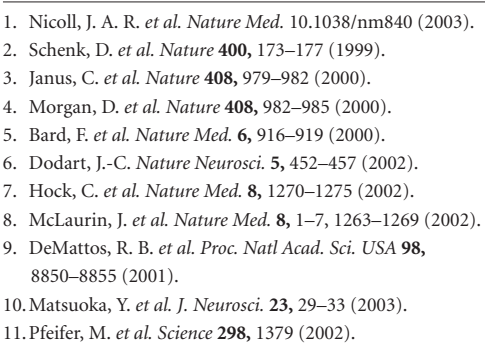

